\title{
SMAC: An interface for investigating sequential multiattribute choices
}

\author{
GAD SAAD \\ Concordia University, Montreal, Quebec, Canada
}

\begin{abstract}
The sequential-sampling approach as applied to multiattribute choices assumes that an individual acquires one piece of attribute information at a time (across the competing alternatives) until sufficient cumulative discrimination has occurred to permit a choice. In such an environment, individuals must not only decide when to stop acquiring additional information but also, whenever additional information is desired, they must determine which attribute to acquire next. The SMAC (Sequential MultiAttribute Choice) interface, described in this paper, was developed as a comprehensive data collection tool relevant to the many distinct stages of this type of sequential choice environment.
\end{abstract}

The classical approach for studying decision making develops normative theories that define what individuals ought to do given a set of economically based rational axioms. In contrast, prescriptive theories propose methods by which decision makers can make good decisions and/or possibly be trained to achieve optimal ones (Bell, Raiffa, \& Tversky, 1988). In contrast to both of the aforementioned approaches, descriptive theories of decision making attempt to capture the processes leading up to a decision. In other words, the descriptive paradigm investigates what individuals actually do when making decisions.

Much of the research in the descriptive tradition has utilized the informational display board (IDB) methodology (see, e.g., Jacoby, Chestnut, \& Fisher, 1978; Payne, 1976; see Ford, Schmitt, Schechtman, Hults, \& Doherty, 1989 , for a review). An IDB is an $m \times n$ matrix whereby the $m$ rows correspond to the $m$ competing alternatives and the $n$ columns represent the $n$ attributes (also referred to as dimensions) defining the alternatives. As such, cell $(i, j)$ in an IDB represents the value of alternative $i$ on attribute $j$. Process-tracing studies that have utilized the IDB technology have typically investigated the decision strategies used in reaching a decision and/or the various characteristics of the search process such as its depth, sequence, content, and latency (see Ford et al., 1989, for an explanation of these measures). Whereas in earlier studies physical representations of an IDB via the use of cardboards and envelopes were employed (see, e.g., Jacoby et al.,

The interface was developed while G.S. was completing his doctoral work at the Johnson Graduate School of Management, Cornell University. G.S. gratefully acknowledges the programming help of Raoul Alejandro Casas and thanks J. Edward Russo for his helpful comments during the development of the interface. Finally, G.S. is grateful to Cornell University and the Social Sciences and Humanities Research Council of Canada for their financial support. The SMAC interface was presented at the Society for Computers in Psychology Conference held in Los Angeles in November 1995. G.S. is in the Faculty of Commerce and Administration at Concordia University. Correspondence should be addressed to G. Saad, Concordia University, 1455 de Maisonneuve Blvd. W. Montreal, PQ, Canada H3G 1M8 (e-mail: gadsaad@vax2.concordia.ca).
1978), more recent work has utilized computerized versions of the IDB (e.g., Payne, Bettman, \& Johnson, 1993).

Computer interfaces have been developed in several other areas of process-tracing decision research. Brucks (1988) developed Search Monitor, an interface that allows an individual to choose both which information to look at and what source to obtain it from. Todd and Benbasat (1991) investigated how different computerized tools for aiding decision making can either facilitate or hinder the use of particular decision rules. Widing and Talarzyk (1993) investigated the objective quality of decisions across several decisional environments and computer-assisted decisional formats. Burke, Harlam, Kahn, \& Lodish (1992) studied whether subjects displayed behavioral differences across real and computer-simulated environments. For a thorough discussion of both the advantages and disadvantages of using computer interfaces in process-tracing research, see Brucks (1988).

Recently, several researchers have departed from the IDB approach, and instead have used a variant of the sequential-sampling model (Wald, 1947) to investigate information search behavior for multiattribute choices (Aschenbrenner, Albert, \& Schmalhofer, 1984; Bockenholt, Albert, Aschenbrenner, \& Schmalhofer, 1991; Saad, 1994; Saad \& Russo, 1995; Schmalhofer, Dietrich, Aschenbrenner, \& Gertzen, 1986). In this approach, it is assumed that a decision maker acquires one piece of attribute information at a time (across all competing alternatives) until sufficient cumulative discrimination/confidence has been achieved to permit a committed choice. For a thorough overview of the sequential-sampling approach in other areas in decision-making research, see Busemeyer and Townsend (1993). This approach is different from the IDB paradigm in three important ways: (1) Contrary to the IDB approach wherein numerous decision rules can be used, the process leading up to a decision is known here; namely, the decision maker acquires attribute information until the desired cumulative threshold of discrimination is reached. (2) The acquisition of a piece of information in an IDB corresponds to the attribute value of a single alternative. 
On the other hand, in a sequential-sampling task, the acquisition of a piece of attribute information corresponds to attribute values across all of the competing alternatives. (3) The direction of search in an IDB can be intradimensional (across two rows, same column), interdimensional (across two columns, same row), or diagonal (different column and different row), whereas in a sequential task, it is solely intradimensional.

Some of the key decisions facing an individual in a sequential-sampling environment include the following: (1) when to stop acquiring additional information (stopping strategy); (2) if additional information is desired, which attribute information to acquire next (acquisition strategy); and (3) if the review of previously acquired information is desired, when such a review should take place and which specific attributes should be reviewed (backtracking strategy). There currently does not exist a computer interface that has been developed to exhaustively address all of the latter issues. Accordingly, the SMAC (Sequential Multi Attribute Choice) computer interface was developed to implement the full sequential decisionmaking environment. The interface runs on a Macintosh computer. It is compatible with many Mactintosh models that use a System 7.0 (or higher) operating system, from the antiquated LC model all the way up to the new Power Macs. The source code was written in ThinkPascal 4.0.1.

\section{Task and Stimulus}

The task consisted of choosing between pairs of apartments to rent for 1 year. Subjects made 15 of these binary choices. The apartments were defined by 25 attributes. Thus, for a given pair of apartments, a subject could request anywhere from 1 to 25 pieces of attribute information prior to making a choice.

This stimulus represents a consequential decision, which subjects (typically university students) will take seriously. In addition, there is little difference in knowledge level across the prospective subjects; hence a potentially confounding covariate is controlled for. This decision stimulus is frequently used in process-tracing research. Ford et al. (1989) found that apartments were used as the decision stimulus in 9 of the 45 studies reviewed. However, whereas the largest number of attributes used in any of the latter 9 studies was 12,25 attributes were used in the SMAC interface.

\section{Description of the SMAC Interface}

Procedure for eliciting the attribute ranks and importance weights. The program begins by alphabetically displaying the 25 attributes. The subjects then go through a Q-Sort procedure to elicit their attribute ranks and weights. Jaccard, Brinberg, and Ackerman (1986) and Stillwell, Seaver, and Edwards (1981) have provided comparisons between various attribute weight and rank elicitation methods. The procedure consists of the following steps: (1) classifying each attribute into one of five categories (very important, important, moderately important, slightly important and unimportant); (2) rank-ordering the attributes within each category; and (3) showing the subjects the 25 attributes in their ranked-order and asking them if they wish to "swap" any. In other words, subjects are given the opportunity to modify any part of the ordered list. The swapping is accomplished by clicking on the two boxes corresponding to the two attributes that the subject wishes to swap. The final rank ordering is then displayed to the subjects in their decreasing order of importance. The most and least important attributes are automatically assigned weights of 100 and 5, respectively, in order to serve as anchors for the 23 remaining attributes. The subject is asked to "work up" the attribute list, assigning numerical weights to reflect the relative importance of the attributes. Clearly, each successive weight must be greater than or equal to the previous one. Failure to meet this condition will result in an auditory beep, with the interface requesting the subject to reenter the weight in question. Note that the above end-point anchors assume a 20-to- 1 ratio of importance between the most and least important attributes.

Attribute acquisition screen. The 25 attributes are alphabetically displayed, and the subject accordingly clicks the box next to the attribute that he/she wishes to acquire next (Figure 1).

Information integration screen. Once an attribute is selected from the attribute acquisition screen, the program transfers to this screen, where the two values corresponding to the requested attribute are displayed. Using the mouse, subjects then update their change in cumulative confidence on a sliding bar. A cumulative confidence of $p$ in favor of Alternative A means that on the basis of the information acquired so far, there is a $(1-p)$ probability that the preference would be reversed in light of all possible information. The lower boundary of this measure of cumulative confidence is 50 (i.e., toss-up between the two alternatives), while the upper boundary is 100 (i.e., zero chance of a reversal of the current preference).

The interface contains an input check for the "directionality" of the entered change in cumulative confidence. For example, if the rents for apartments $A$ and B are $\$ 500$ and $\$ 700$, respectively, the interface assumes, ceteris paribus, that the former will be preferred on the basis of that attribute. Thus, if the entered change favors Alternative $B$, the interface will flag the user by asking whether he/she is certain of his/her response. The subject can then either change it (in case of error) or simply proceed.

Once the change in cumulative confidence is entered, the subject decides between requesting an additional piece of information (in which case control is transferred back to the attribute acquisition screen) or choosing the leading apartment (Figure 2).

Past information screens. This is an optional set of screens that can be accessed from the information integration screen. It allows subjects to review previously acquired information. Once this option is chosen, subjects see a listing of the attributes in their ranked order. Furthermore, they can access an additional subscreen that displays a tracking of the changes in cumulative confidence throughout the current choice. Figure 3 provides a flowchart summary of SMAC's interactive flow. 


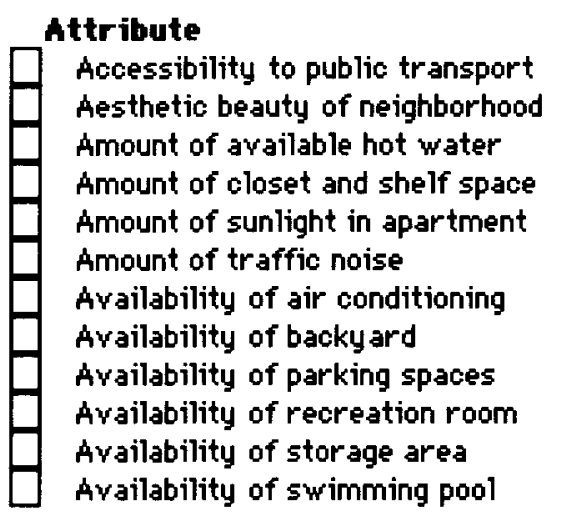

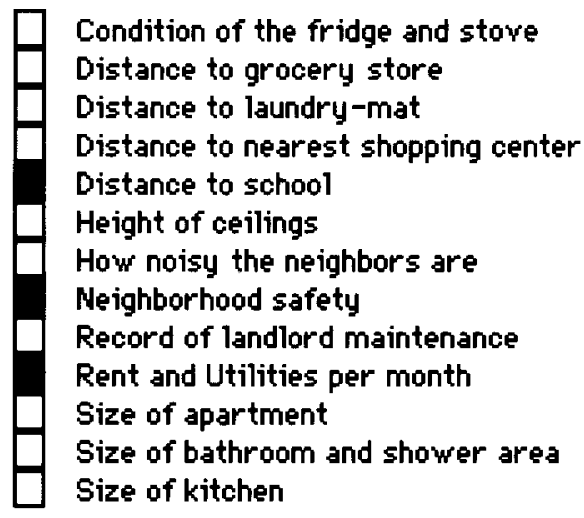

Figure 1. The attribute acquisition screen. The 25 attributes are shown in alphabetical order. Blackened boxes correspond to attributes that have already been acquired. This figure was originally presented in Saad (1994, p. 77). Reprinted with permission.

\section{Collected Data}

The interface collects an extensive amount of processtracing information, which is stored in three text files: summary, acquisition, and past_info. The contents of each of the latter output files are described below.

The summary output file contains the following:

1. The elicited attribute ranks and weights, listed in decreasing order of importance.

2. For each of the 15 binary choices,

a. The number of attributes acquired prior to making a choice.

b. The cumulative confidence in the leading alternative when a choice is made.

c. The total time spent (in seconds) on each of the three decisional stages, namely the attribute acquisition, the information integration, and the review of previously acquired information screens.

3. The total number of times that each attribute is acquired across the 15 binary choices.

The acquisition output file contains the following: For each of the 15 binary choices,

a. A listing of the attribute acquisition order, which includes the acquired attributes' ranks and importance weights.

b. For each of the acquired attributes in a given choice, the number of seconds spent in the attribute acquisition and information integration stages.

The past_info output file contains the following:

1 . The change in cumulative confidence for each acquired attribute.

2. For each of the 15 binary choices,

a. Whether the past_info option was used and if so the order in which the attributes were reviewed and their respective ranks and weights.

b. A "flagging" whenever the tracking curve is looked at.

c. A "flagging" whenever a request is made to review an attribute that has yet to be acquired. Both the attribute's rank and weight are also shown.

\section{Construction of the Trials}

The interface uses two data structures in determining which attribute values subjects will view once they have requested an additional piece of attribute information. Each is separately described below.

Data structure 1. A trial configuration consists of specifying, for each of the 25 attributes that might be acquired in a given choice, the size of the attribute difference between the two competing alternatives and whom it favored. Attribute differences are subjectively classified as small, medium, and large. Thus, each of the $15 \mathrm{bi}-$ nary choices has a specific configuration of attribute order and attribute differences. For example, denoting small, medium, and large attribute differences by $\mathrm{s}, \mathrm{m}$, and 1 , respectively, the configuration of the seven most important attributes for a given trial might look as follows: $+\mathrm{l}$, $-\mathrm{s},-\mathrm{m},+1,-1,+\mathrm{m},+\mathrm{s}$. The \pm sign determines which of the two alternatives is superior on an attribute, whereas the attribute differences are specified by $\mathrm{s}, \mathrm{m}$, or $\mathrm{l}$. In the above configuration, the most important attribute will yield a large attribute difference favoring one alternative whereas the second most important will result in a small difference favoring the other alternative. The trial configurations are stored in an array of records. The rows of the array correspond to the 15 binary choices whereas the columns represent the 25 attributes in descending order of importance. In other words, cell $(i, j)$ of the array corresponds to the configuration of a subject's $j$ th most important attribute in the $i$ th binary choice. Note that the latter data structure is generic in that it specifies the trial configurations irrespective of the subject-specific attribute rankings.

Data structure 2. A second data structure, consisting of an array of pointers and linked lists, specifies the actual attribute values for a given attribute difference. Each of the 25 attributes has three linked lists, corresponding to the three sets of small, medium, and large attribute differences. Each of the records within a linked list specifies a pair of attribute values. A pointer "moves along" 
SIZE OF APARTMENT (in square feet)

Apartment $A$

330
Apartment B

270

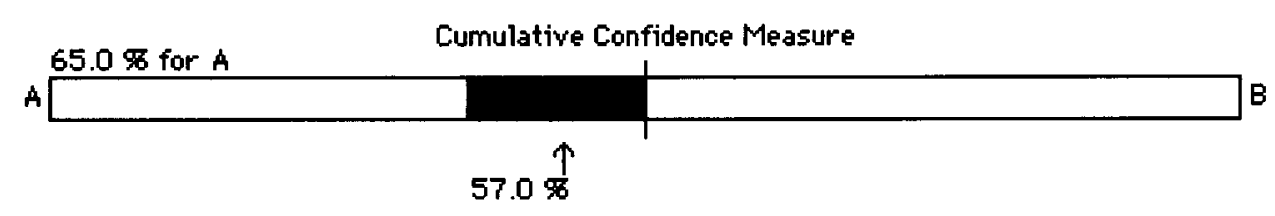

Next Piece of Information

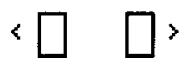

Past Information

Choose Apartment

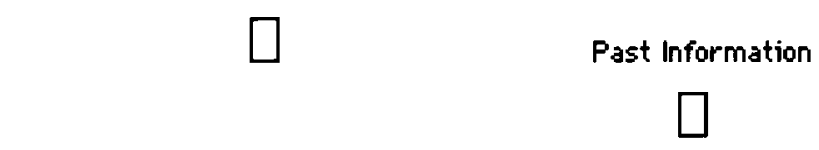

Messages

Decision \#
Piece of info

Figure 2. The information integration screen. The $57 \%$ marker represents the cumulative confidence value prior to the subject's looking at this fourth piece of information. In this example, the subject has moved from $57 \%$ to $\mathbf{6 5 \%}$ (in favor of alternative $A$ ) as a result of this newly acquired information. This figure was originally presented in Saad (1994, p. 78). Reprinted with permission.

each of the linked lists, generating new attribute values for a given attribute difference. If a pointer reaches the end of a linked list, it "loops" back to its start. Clearly, for those attributes that are either binary or ternary (i.e., that consist of two or three levels, respectively), the same linked list is repeated thrice (for binary variables) and twice (for ternary variables).

\section{General Discussion}

Given the novelty of the sequential-sampling approach in the context of risk-free multiattribute choices, few interfaces have been developed for such an environment. Of the existing interfaces, none is as comprehensive as the SMAC system described here. The latter is the sole interface that addresses this many stages of the sequential process, including the stopping, acquisition, and backtracking stages. The interface has already been used to investigate individuals' stopping strategies (see Saad \& Russo, 1995, in which an earlier version of SMAC was used; see also Saad, 1994), the adaptive use of such strategies (Saad, 1994), the relationship between acquisition order and attribute ranks (Saad, 1995), and various other facets of back-tracking behavior (Saad, 1995). A substantive research program is currently under way that will make further use of the SMAC system. Some of the issues to be addressed include the investigation of situational and individual factors affecting the use of particular stopping strategies, the identification of new factors affecting the order of attribute acquisitions, and the study of motivational distortions and violations of rationality in sequential choice.

\section{Future Improvements to SMAC}

Currently, the interface is static in that it works only for choices between pairs of apartments defined by 25 attributes. A more dynamic version of the interface would allow the experimenter to interact with a shell, prior to the running of an experiment, allowing him/her to construct other decision stimuli (e.g., choices between pairs of universities defined by 20 attributes). In a similar vein, the attribute acquisition screen does not presently include any factors that might otherwise affect the order of attribute acquisitions. For example, one could implement acquisition costs by placing a set of time delays next to the listed attributes (Brucks, 1988). Thus, subjects would have to take into account both the attribute ranks and their associated costs when deciding which attribute to acquire next.

The first two screens in the past information option allow only for a passive review of the previously acquired information. In other words, for a given piece of attribute information, one cannot alter a previously entered change in cumulative confidence. Svenson (1992) proposed that individuals apply postdecisional consolidation processes to defend a chosen alternative against possible threats. Two such operations include bolstering (discounting) the impact of attributes on which the chosen alternative is superior (inferior). Clearly, providing subjects with the opportunity to make changes from within the past information option would allow for an "on-line" test of Svenson's theory.

In conclusion, a dynamic and interactive shell would permit the experimenter to easily alter some if not all of 


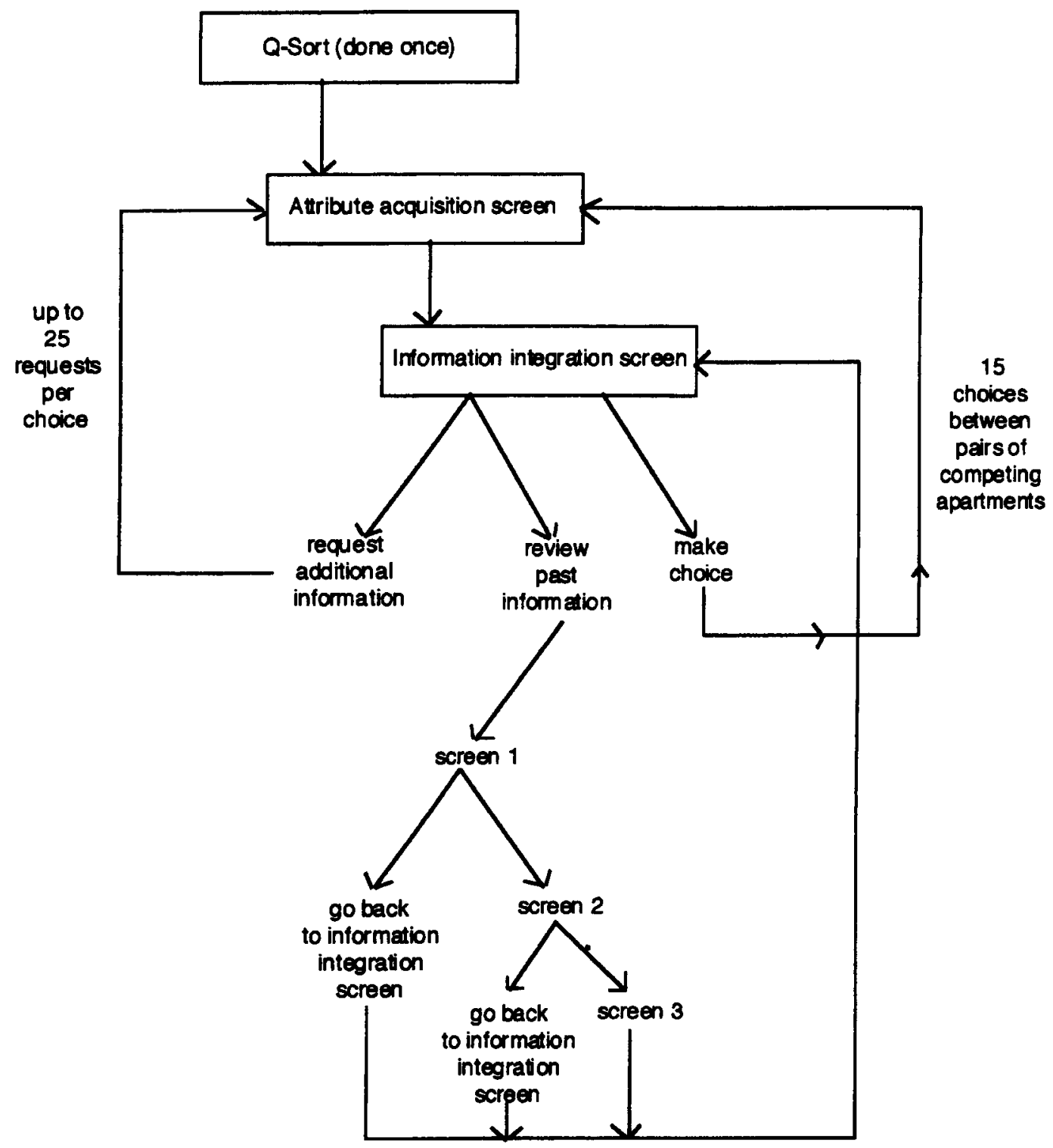

Figure 3. Flowchart of SMAC's interaction flow.

the relevant experimental task parameters, saving him/her from having to constantly make source code modifications to the interface.

\section{REFERENCES}

Aschenbrenner, K. M., Albert, D., \& Schmalhofer, F. (1984). Stochastic choice heuristics. Acta Psychologica, 56, 153-166.

Bell, D. E., Raiffa, H., \& TVerSKY, A. (1988). Descriptive, normative, and prescriptive interactions in decision making. In D. E. Bell, H. Raiffa, \& A. Tversky (Eds.), Decision making: Descriptive, normative, and prescriptive interactions (pp. 9-30). New York: Cambridge University Press.

Bockenholt, U., Albert, D., Aschenbrenner, M., \& SchmalHOFER, F. (1991). The effects of attractiveness, dominance, and attribute differences on information acquisition in multiattribute binary choice. Organizational Behavior \& Human Decision Processes, 49, 258-281.
BrucKs, M. (1988). Search monitor: An approach for computercontrolled experiments involving consumer information search. Journal of Consumer Research, 15, 117-121.

Burke, R. R., Harlam, B. A., KahN, B. E., \& Lodish, L. M. (1992). Comparing dynamic consumer choice in real and computer-simulated environments. Journal of Consumer Research, 19, 71-82.

Busemeyer, J. R., \& TownSEnd, J. T. (1993). Decision field theory: A dynamic-cognitive approach to decision making in an uncertain environment. Psychological Review, 100, 432-459.

Ford, J. K., Schmitt, N., Schechtman, S. L., Hults, B. M., \& DoHERTY, M. L. (1989). Process tracing methods: Contributions, problems, and neglected research questions. Organizational Behavior \& Human Decision Processes, 43, 75-117.

JaCCARD, J., Brinberg, D., \& ACKerman, L. J. (1986). Assessing attribute importance: A comparison of six methods. Journal of Consumer Research, 12, 463-468.

JaCoby, J., Chestnut, R. W., \& Fisher, W. A. (1978). A behavioral process approach to information acquisition in nondurable purchasing. Journal of Marketing Research, 15, 532-544. 
PAYNe, J. (1976). Task complexity and contingent processing in decision making: An information search and protocol analysis. Organizational Behavior \& Human Performance, 16, 366-387.

Payne, J. W., Bettman, J. R., \& Johnson, E. J. (1993). The adaptive decision maker. Cambridge: Cambridge University Press.

SAAD, G. (1994). The adaptive use of stopping policies in sequential consumer choice. Unpublished doctoral dissertation, Johnson Graduate School of Management, Cornell University.

SAAD, G. (1995). Attribute acquisition and backtracking behavior in sequential choice. Unpublished manuscript, Concordia University, Montreal.

SAAD, G., \& Russo, J. E. (1995). Stopping criteria in sequential choice. Unpublished manuscript, Concordia University, Montreal.

Schmalhofer, F., Dietrich, A., Aschenbrenner, K. M., \& GertZEN, H. (1986). Process traces of binary choices: Evidence for selective and adaptive decision heuristics. Quarterly Journal of Experimental Psychology, 38A, 59-76.

Stillwel., W. G., Seaver, D. A., \& Edwards, W. (1981). A compar- ison of weight approximation techniques in multiattribute utility decision making. Organizational Behavior \& Human Performance, 28, 62-77.

Svenson, O. (1992). Differentiation and consolidation theory of human decision making: A frame of reference for the study of pre- and postdecision processes. Acta Psychologica, 80, 143-168.

TodD, P., \& Benbasat, I. (1991). An experimental investigation of the impact of computer based decision aids on decision making strategies. Information Systems Research, 2, 87-115.

WALD, A. (1947). Sequential analysis. New York: Wiley.

WIDING, R. E., \& TALARZYK, W. W. (1993). Electronic information systems for consumers: An evaluation of computer-assisted formats in multiple decision environments. Journal of Marketing Research, $\mathbf{3 0}$, 125-141.

(Manuscript received November 28, 1995; revision accepted for publication February 5, 1996.) 